\title{
From the 40s to the 70s - the future of LCA in the ISO 14000 family
}

\author{
Matthias Finkbeiner
}

Received: 23 August 2012 /Accepted: 23 August 2012 /Published online: 5 September 2012

(C) Springer-Verlag 2012

\section{Background}

If you are curious about the $40 \mathrm{~s}$ and $70 \mathrm{~s}$, I can give you the hint that they do not represent year dates here. This is not an editorial about history. It is an update on the current status of life cycle assessment (LCA) standards and their offspring as part of the ISO 14000 family. The mystery of the 40s and 70s will be unraveled a bit later. Let's get down first to brass tacks!

International standards for LCA were developed since the 1990s by ISO Technical Committee 207 as part of their ISO 14000 family of environmental management standards. They were of utmost importance for the broad acceptance of LCA worldwide. They are, until today, the one and only relevant international standard documents on LCA which are broadly referenced by users and other standardization processes. They represent the constitution of LCA.

Based on the core standards "ISO 14040-Environmental Management-Life Cycle Assessment-Principles and Framework (ISO 14040 2006)" and "ISO 14044Environmental Management-Life Cycle AssessmentRequirements and Guidelines (ISO 14044 2006)," recent developments led to "new" approaches and some spin-off standards. They cover issues like the:

- "Single-issue LCAs" like carbon footprinting (ISO 14067) or water footprinting (ISO 14046)

- "Beyond environment LCAs" like life cycle costing, social LCA, and eco-efficiency assessments (ISO 14045) or even life cycle sustainability assessments

- "Beyond product LCAs" like scope 3-type LCAs of organizations (ISO 14072) or sector-based IO-LCAs, and

\footnotetext{
M. Finkbeiner $(\triangle)$

Department of Environmental Technology,

Chair of Sustainable Engineering, Technical University Berlin,

Office Z1, Strasse des 17. Juni 135,

10623 Berlin, Germany

e-mail: matthias.finkbeiner@tu-berlin.de
}

- "Beyond quantification LCAs" like type III environmental product declarations (ISO 14025 2006) or other types of environmental labels and claims

The last more comprehensive paper on ISO standards in this journal was published in 2006, just after the last revision of the core standards (Finkbeiner et al. 2006). Some standardization aspects with regard to carbon footprinting were discussed in the editorial to announce the carbon footprint section of this journal (Finkbeiner 2009), and very recently, Klöpffer discussed the critical review of compliance with the LCA standards (Klöpffer 2012a). However, a more comprehensive overview of recent developments in the standardization area has been lacking, and it is the main target of this editorial to bring you up to date in this respect. This will be done by a report and discussion of the results of the recent ISO TC207 plenary meeting in Bangkok. A more comprehensive coverage of the history, present, and future of the ISO standardization of LCA is foreseen in volume I of the LCA encyclopedia which was recently announced in this journal (Klöpffer 2012b).

\section{Status and results from the last plenary meeting of ISO TC 207}

The last plenary meeting of ISO TC 207 was held end of June this year in Bangkok. About 350 delegates from over 40 countries were dealing with the different standards in the ISO 14000 family. With regard to life cycle-based standards, the meetings and negotiations on the upcoming carbon footprint and water footprint standards generated the most interest. In both cases, working group meetings were attended at times by some 100 delegates.

The carbon footprint project is much older than the water footprint project. The work on this topic is done by ISO/ TC207/SC7 on Greenhouse Gas Management and is 
supposed to lead to ISO 14067 "Carbon Footprint of Products-Requirements and Guidelines for Quantification and Communication" (ISO/DIS 14067 2012). According to the introduction of the current draft international standard, "this International Standard is based on existing ISO standards, e.g., ISO 14020, ISO 14025, ISO 14040 and ISO 14044 and aims to set more specific requirements for the quantification and communication of carbon footprints of products (CFP)." While the current draft of the standard has grown to a lengthy document of over 50 pages, most of its content is rather a repetition of the content of previous standards. For the quantification part, a lot of ISO 14044 content is copied into ISO 14067. For the average reader of this journal, ISO 14067 will bring neither much news nor a broad range of specific requirements. As a matter of fact, the additional CFPspecific requirements for quantification would fit in a handful of pages. While such a "delta" standard would have been more efficient, the working group wanted to develop a "standalone" document. This was - amongst others (e.g., merging of the originally separate quantification and communication parts, lack of knowledge on underlying standards, lack of team spirit and process quality) - one of the reasons why the overall standardization process took and still takes much longer than it was supposed to be.

Originally, the publication of the standard was due in March 2011. If the final steps go well, the publication of the document can hopefully be expected in early 2013 . However, for the finalization of the standard, an element of risk remains, because the different committee drafts and draft international standards have so far been rejected several times in the voting of the national standardization bodies. Such a repeated rejection of standard documents has never happened before in TC 207, because the consensus principle in developing the documents usually leads to positive voting results.

The work on water footprinting is still on track-even though the standardization process is technically more challenging than carbon footprint. Due to the state of the art in water footprinting methodologies, it is premature to expect a standard that will fix the method to do it. The first version of the standard is about agreeing on the relevant terminology and some key methodological issues and concepts. One of these issues is the discussion of volumetric vs. impactoriented water footprint methods (Berger and Finkbeiner 2012). While, for example, the method of the Water Footprint Network, which deserves credit for bringing the issue on the agenda, uses an inventory of water volumes, it is nowadays broadly accepted that this is scientifically not sufficient to address the issue of water scarcity. This is acknowledged by ISO 14046 which currently defines a water footprint clearly on the impact level as "parameter(s) that quantify(ies) the potential environmental impacts related to water" (ISO/CD.1 14046 2012).
According to the current committee draft document (ISO/ CD.1 14046 2012), the scope is defined as "specifying principles, requirements and guidelines to assess and report the water footprints of products, processes and organizations based on life cycle assessment (LCA). The standard provides requirements and guidance for calculating and reporting a water footprint as a stand-alone assessment or as part of a more comprehensive environmental assessment. The water footprint is calculated as one impact indicator result or multiple impact indicator results." Due to the large participation in the work and its relevance, the prediction of the publication date involves uncertainties. However, based on the current project plan, the publication of the document is expected for 2014.

Next to these two high-profile projects, there were announcements of newly published standards in the field of LCA, newly started standardization processes, and discussions on the need for a revision of the core standards. Newly published are three documents: the document called "ISO/TR 14047:2012 Environmental Management—Life Cycle Assessment-Illustrative Examples on How to Apply ISO 14044 to Impact Assessment" (ISO/TR 14047 2012) was published in an editorially improved version of the original document from 2003-mainly to make it consistent with the current versions of ISO 14040 and ISO 14044. As there was no technical update during the revision, several of the examples given do not necessarily represent the latest state of the art in impact assessment anymore. In the same way, an editorially updated version of "ISO/TR 14049:2012 Environmental Management-Life Cycle Assessment-Illustrative Examples on How to Apply ISO 14044 to Goal and Scope Definition and Inventory Analysis" (ISO/TR 14049 2012) was published.

More novelty comes with the standard "ISO 14045:2012 Environmental Management-Eco Efficiency Assessment of Product Systems - Principles, Requirements and Guidelines" which represents an important step due to a broader focus beyond environmental issues only (ISO 14045 2012). "Ecoefficiency assessment is a quantitative management tool which enables the consideration of life cycle environmental impacts of a product system alongside its product system value. Within eco-efficiency assessment, environmental impacts are evaluated using Life Cycle Assessment (LCA). The value of the product system may be chosen to reflect, for example, its resource, production, delivery or use efficiency, or a combination of these. The value may be expressed in monetary terms or other value aspects. The key objectives of this International Standard are to establish clear terminology and a common methodological framework for eco-efficiency assessment; to enable the practical use of eco-efficiency assessment for a wide range of product (including service) systems; to provide clear guidance on the interpretation of eco-efficiency assessment results and to encourage the 
transparent, accurate and informative reporting of ecoefficiency assessment results" (ISO 14045 2012).

With regard to the Technical Specification "Environmental Management-Life Cycle Assessment—Data Documentation Format" (ISO/TS 14048 2002), it was announced that this document currently undergoes the systematic review procedure. This means that the national member bodies currently vote, whether this document shall be withdrawn (in case of lacking market need), confirmed (in case of still existing market need and technical validity), or revised (technical improvements needed). There was an intervention from Sweden which proposed to work on a revision of the document, but the result of the ongoing ballot will finally determine the direction to take.

Two work items are already active and had their first formal working group meetings in Bangkok. The proposal to develop a Technical Specification ISO 14071 "Environmental Management—Life Cycle Assessment-Requirements and Guidelines for Critical Review Processes and Reviewer Competencies" was motivated from the discussion on conformity assessment of, e.g., the carbon footprint standards or upcoming labeling initiatives. As part of these processes, different interested parties proposed different conformity assessment schemes including critical review according to ISO 14040 and ISO 14044, verification according to ISO 14025, but also the bureaucratic accountant approach according to greenhouse gas verification. The critical review approach was very successful within the LCA community. One of the key success factors is that the system does not operate an accreditation scheme which tries to ensure quality by bureaucracy and in which verification bodies that can afford the overhead cost then send some inexperienced individuals actually doing the job. The critical review system ensured quality by making the individual reviewer personally accountable for the work and spending the resources on content rather than paperwork.

While this is generally accepted in the LCA world, the critical review system had a challenge to be argued "against" the bloated verification documents of other schemes. In order to document the well-established critical review practice in a more formal way, the intention of this coming international technical specification is to provide requirements and guidelines for conducting a critical review and the competencies required. The target is to provide a crisp and lean specification that documents the established best practice for performing critical reviews. The publication of the document is expected in 2014.

The next standardization project deals with LCA for organizations. The benefits and the potential of the life cycle approach are not limited to an application on products. While it is true that the LCA methodology was originally developed for products, its application on the organizational level is getting more and more relevant. The discussions on carbon footprinting of companies including their upstream and downstream supply chains-the so-called "scope 3" (GHG-
Protocol 2012) revealed that these "life cycle" emissions can contribute significantly to the organizational footprint. The currently applied assessments mostly concentrate on a single aspect like carbon or water footprints. The purpose of this new standard is to present a general and comprehensive approach by adapting LCA methodology on organizations. The document ISO 14072 is supposed to be a Technical Specification (TS) called "Life Cycle AssessmentAdditional Requirements and Guidelines for Organizations." The main goal is to provide additional guidance to organizations for an easier and more effective application of ISO 14040 and ISO 14044 on the organizational level including the advantages that LCA may bring to organizations. The publication of the document is expected in 2014.

By now, the attentive reader got the point about the $40 \mathrm{~s}$ and the 70s. For all these years, we were used to talk about the "forty series" when we referred to the international standards of LCA. Now, we have to come up with something new, because we ran out of remaining numbers in this series. The LCA Committee of ISO TC207 (Subcommittee 5 ) is the first that exhausted the original number block. The new series we got is the 70s. Special thanks go to the ISO Central Secretariat for giving us the 14070s, because originally, the new projects described above were supposed to get more obscure numbers. So, we have now ISO 14071 and ISO 14072 in the pipeline. Some may wonder why they start with 14071 and not with ISO 14070. The ISO Central Secretariat wanted to reserve that number for a "high-profile" standard, whatever this will be. Suggestions are welcome.

Last, but not least, there were some more fundamental discussions on the need and potential areas for a revision of the core standards ISO 14040 and ISO 14044. Over the last two plenary meetings, national member bodies were asked for their position on this, and proposals for changing or adding certain aspects of the standards were collected and evaluated. The proposals covered a broad range of issues from rather editorial changes, over formal changes like the merger of the current two standards into one single document to more substantial technical changes reflecting topics that are currently discussed in the scientific LCA community or the footprinting arena. I will not go into details here, because picking some particular issues from the long list proposed might give wrong signals. The proposed topics for a potential revision were evaluated against the criteria risk vs. opportunity, priority level, added value, and level of consensus. At the end of the day, none of the proposals received an evaluation that would justify an immediate revision. Some member bodies also argued that the current standards have to remain in place as a stable anchor until "all these footprinting standards are done." From a standardization perspective, there is some substance in this argument, because an existing standard has to be adhered to. It 
was not an easy task to tame some of the carbon footprint cowboys. Without a solid ISO 14040 and 14044 in place to which they could be referred, this would have been even more difficult.

However, despite a broad consensus not to revise the standards right now, there was also an indication that a modest revision of the standards in a medium term might be on the horizon. If the experiences gained in the footprinting standards and some of the proposals brought to the table in Bangkok have further matured, a consensus on a revision of a specific set of items might be reached within a couple of years.

\section{Conclusion and future perspectives}

Standards play an important role in business and everyday life. They represent a consensus on good practice and state of the art. This applies to all kinds of technical topicsincluding LCA. The establishment of the international standards of LCA (ISO 14040/70 series) was of utmost importance for the broad acceptance of LCA all around the world and by all stakeholders. The standards contributed significantly to the transition of LCA from an academic toy or misused greenwashing machine towards a serious, robust, and professional tool to support decision-making in public and private organizations.

They represent the constitution of LCA and should therefore be respected and protected by everyone. It is fair to ask for more specific stipulations in future versions, if global consensus evolves on such issues. If such a consensus does not exist, we have to be aware that asking for more sometimes leads to getting less than we already have-half a loaf is better than no bread.

Some future activities have already been highlighted above. They represent fairly well the future direction that the author anticipates at this point in time. We will have some additional standards that specify particular parts of LCA methodology (e.g., critical review, LCA on organizations), we will have some further standards on simplified LCA versions (e.g., carbon or water footprinting), and we will expand the environmental focus towards all three sustainability dimensions (resource efficiency, life cycle costing, social LCA, life cycle sustainability assessment). All these developments shall support the credible and robust use of LCA for real-world decision-making in the sense of life cycle management and life cycle sustainability management (Finkbeiner 2011). In systems that support participation of citizens and democracy, this requires commonly accepted rules. ISO 14040 and ISO 14044 have become the commonly accepted rules for LCA. It is by no means a guarantee for sustainable development, but it makes it more likely.
Acknowledgments I would like to thank Melanie Raimbault (France) for her outstanding work, support, and good times during the past 8 years as Secretary of ISO TC207/SC5, and I would like to welcome Sandrine Delalieux (France) as new SC5 Secretary. Special thanks have to go to Reginald Tan (Singapore) as distinguished SC5co-chair. I also have to give kudos to the hardworking convenors and secretaries of the recent working and task groups, namely Bengt Steen (Sweden), Reginald Tan (Singapore), and Karin Bagge (Sweden) for WG7 on Eco-efficiency Assessment, Sebastien Humbert (Switzerland), Nydia Suppen (Mexico), and Marcel Schulze (Switzerland) for WG8 on Water Footprinting, Sergio Galeano (USA) for the Task Force to edit ISO/TR 14047 and ISO/TR 14049, and Phillippe Osset (France), Chen Liang (China), and Melanie Raimbault (France) for WGs 9/10 on Critical Review/LCA for Organizations, respectively. Last but not least, sincere thanks to all SC5 members and experts for their contributions and spirit.

\section{References}

Berger M, Finkbeiner M (2012) Methodological challenges in volumetric and impact-oriented water footprints. J Ind Ecol. doi:10.1111/j.1530-9290.2012.00495.x

Finkbeiner M (2009) Carbon footprinting - opportunities and threats. Int J Life Cycle Assess 14(2):91-94

Finkbeiner M (2011) Towards life cycle sustainability management. Springer, Berlin, ISBN 978-94-007-1898-2

Finkbeiner M, Inaba A, Tan RBH, Christiansen K, Klüppel HJ (2006) The new international standards for life cycle assessment: ISO 14040 and ISO 14044. Int J Life Cycle Assess 11(2):80-85

GHG Protocol (2012) Corporate value chain (scope 3) accounting and reporting standard, 2011, http://www.ghgprotocol.org/ files/ghgp/Corporate $\% 20$ Value $\% 20$ Chain $\% 20$ (Scope $\% 203) \% 20$ Accounting\%20and\%20Reporting\%20Standard.pdf, Accessed 17 Aug. 2012

ISO 14025 (2006) Environmental labels and declarations, type IIIenvironmental declarations - principles and procedures. Geneva, Switzerland

ISO 14040 (2006) Environmental management—life cycle assessment - principles and framework. Geneva, Switzerland

ISO 14044 (2006) Environmental management — life cycle assessment -requirements and guidelines. Geneva, Switzerland

ISO 14045 (2012) Environmental management-eco-efficiency assessment of product systems-principles, requirements and guidelines. Geneva, Switzerland

ISO 14046.CD.1 (2012) Water footprint-requirements and guidelines. Geneva, Switzerland

ISO/TR 14047 (2012) Environmental management-life cycle assessment - illustrative examples on how to apply ISO 14044 to impact assessment situations. Geneva, Switzerland

ISO/TS 14048 (2002) Environmental management-life cycle assessment-data documentation format. Geneva, Switzerland

ISO/TR 14049 (2012) Environmental management-life cycle assessment-illustrative examples on how to apply ISO 14044 to goal and scope definition and inventory analysis. Geneva, Switzerland

ISO/DIS 14067 (2012) Carbon footprint of products-requirements and guidelines for quantification and communication. Geneva, Switzerland

Klöpffer W (2012a) The critical review of life cycle assessment studies according to ISO 14040 and 14044 - origin, purpose and practical performance. Int J Life Cycle Assess. doi:10.1007/s11367-0120426-7

Klöpffer W (2012b) Thank you, Almut! Int J Life Cycle Assess 17 (8):947-948 